\title{
1.4 Volitional or autonomous behavior management: theoretical aspects for pedagogical practice
}

Nowadays one of the key problems in the educational process is managing the behavior of a person. This problem is insufficiently disclosed both in theory and practice of pedagogy (especially when it comes to the development of a strong-willed, autonomous personality). This problem is associated with the phenomenon of the concept of spiritual and moral development of the individual, where the basis is the autonomous spirit, volitional choice, pragmatic action, which is consistent with the society values.

Recently, the study of various aspects of this problem has appeared in the pedagogical literature. Thus, there appear such positions as teacher autonomy in its relationship with self-efficacy (Soobin Choi, Xinyi Mao, 2021) [36], the implementation of autonomy in pedagogical practice (Xuan Nhat Chi Mai Nguyen, Ian Walkinshaw, 2018) [37], the ability of the learner to autonomous and critical thinking (José FO Granjo, Maria G. Rasteiro (2020) [38], social, individual and cultural dimensions of autonomy in teaching (Biljana Ivanovska, 2015) [39], autonomy with respect to teaching methodology, instructional materials, course content, assessment, lesson planning (Cirocki, Andrzej, Anam, Syafi'ul, 2021) [40], autonomy as a central component of learning, teaching (Diane Railton, Paul Watson, 2005) [41], the impact of autonomy on student well-being (Arielle Bonneville-Roussy, Emese Hruska, and Hayley Trower, 2020) [42] and others.

We find an important conclusion about the support of autonomy in the work of authors such as Arielle Bonneville-Roussy, Emese Hruska, and Hayley Trower: «It is important to emphasize that autonomy support relates to promoting autonomy within a given structured environment. That is, autonomy support is not synonymous to a laissez-faire or hands-off form of teaching. Autonomy-supportive teachers recognize that students are in charge of their own learning but also acknowledge that to master skills, certain tasks need to be accomplished within a given structure accompanied by 
guidance. That is, autonomy-supportive teachers provide both choice and structure» [7, p. 99].

From our point of view, to solve the problem of managing the behavior of a person, a scientific concept of spiritual and moral development is needed, which will take into account the position of goal-setting, the basic principles of self-government, the main forms of expression of individual autonomy of the individual. This problem changes our understanding of the inner work of the individual on himself and the understanding of the laws of upbringing for further pedagogical practice.

It is worth noting that there is a wide variety of approaches, ideas, concepts in pedagogy that allow us to consider the positions of behavior management. However, despite their application in the cultural and educational practices of the teacher, there are many ambiguous decisions that do not allow to fully understand how to develop the personality better, how to improve the pedagogical process.

One of the central approaches used in the pedagogical process is activity-based approach. It gives an opportunity to consider the actions of an individual, his intentions and motivation, the impact of various forces (both external and internal) on the development of the individual. In this case, we can also consider the concept of authorship of an individual's actions in the direction of his spiritual and moral development. At the same time, it should be noted that an action must be conscious for spiritual and moral development, that is, it should be one that has passed through the feeling and mind of the individual, through his/her experiences and reflection.

There is no doubt, the pedagogy of action, which includes the disclosure of the processes associated with the formation of volitional and autonomous behavior, is based on the idea of a number of religious (divine will) and philosophical (reasonable desire, will as the initial basis of human activity) studies, psychological problems (autogenetic and heterogenetic theories of will, volitional influences and efforts, etc.).

Currently, they develop methods in pedagogical researches that train the ability of a person to achieve a goal, that is, goal-setting in this regard is a primary element which must be taken into account in order to solve problems of spiritual and moral development. It should be noted that this process, seemingly imperceptible, is essential 
for the pedagogical space in which it unfolds. Thus, pointing to the issues of selfmanagement of the individual, we mean that the development of the problem of educating volitional or autonomous behavior becomes obvious.

The difference between strong-willed behavior and behavior based on instinct can be seen in the comparative characteristics of game situations in team sports. In games, we can observe the coach's conscious work with the players when building a plan to achieve the goal, developing and adjustg the program of actions that are aimed at the result. We can also observe the chaotic actions of a spontaneously assembled team that has no plan, no organization, no structure and no idea of the rules of the game. In a spontaneous team, as in a spontaneous society, there are main characteristics such as a conflict, misunderstanding, lack of the main goal and practical guidance, and, accordingly, there is no result. We can observe in this case the manifestations of ataraxia (the opposite of will), i.e., weak will, intemperance.

However, if we are talking about the spiritual and moral development of the individual, then the team of players is of the person's close environment, and much in the development of the growing personality depends on whose goals are pursued in behavior. It is difficult to say with certainty that the close environment of the growing personality as a whole has the goals of spiritual and moral development, or that the autonomy of the spirit is clearly revealed in their behavior. Although we can note that in some communities there may be a focus, a certain dynamic of development aimed at improving the quality of life in general, but most decisions, even in organized societies, are more focused on improving the material rather than the spiritual side of life.

It is noteworthy that in the spiritual and moral development of the individual, the goals are not always conscious and clearly formulated, and the results are not fully thought out and, thus, are not always achievable. In this regard, for the formation of strong-willed or autonomous behavior of a person, it is necessary to develop and implement special technologies and techniques, since each person is unique in its characteristics and has a certain experience, knowledge and skills. Also, the personality develops not separately, but in a specific culture, which has an impact on the formation 
of sociability, stereotypes, and generally accepted views. By agreeing with the rules and laws of a particular society, the individual partially gives up his autonomy, obeying ethical considerations. However, the acceptance of the ethical considerations of society should not be confused with the weakening of the will, which leads to a weakening of the desire, a loss of responsibility for the earlier decision to act.

In order to develop volitional or autonomous behavior of the individual, it is necessary to create an environment that will be focused on all subjects of the process of spiritual and moral development, which makes special demands. The management of autonomous behavior can be described in various situations of interaction in the systems «a teacher and a student», «a student and a student», «a teacher, a parent and a student». In such situations, you can see new opportunities for building a conscious plan for your own development, which will mark the beginning of the autonomy. If each participant in the educational process consciously creates a plan, and implements it in his life, taking into account personal motives and the ability to act, then autonomy will acquire an individual dimension. It is also necessary to avoid extremes - the autonomy of the spirit, independence and critical judgment has nothing to do with asociality or antisociality. We are talking about a mandatory component of autonomous behavior - mutual support of the Other, which provides the disclosure of the cultural and educational space for the development of autonomous behavior.

Understanding the processes of controlling volitional or autonomous behavior is an important issue for both parents and teachers. Most teachers can offer recommendations that will help develop respect for the student, his individual characteristics, which include both scientifically based psychological and pedagogical facts, and the reality of the manifestation of feelings and experiences observed by the teacher for a certain period of time.

At the same time, we can note that most teachers understand the development of volitional processes of behavior and are able to manage autonomous students, but teachers themselves are not always sure of their own autonomy when they have to make a decision (especially in an environment where interpersonal conflicts break out). Such violations acquire an unnatural character, since the demonstration of free will can be 
taught by a person who is autonomous himself, and if there are exceptions, this makes a negative impression on the trainees.

That is why it is important to have certain laws of relations between autonomous personalities, which are established through dialogical practices. The array of opportunities for dialogic communication that accompanies relationships in the systems of students and their parents, students and teachers, students and students, shows what new things can be learned about yourself in the spiritual dimension.

It can be noted that a high level of autonomous behavior will be revealed both individually and in certain communities, and the demonstration of autonomy is one of the first steps that lead to an increase of this level. The development of a high level of autonomous behavior is possible in different environments of pedagogical culture, and here one of the important factors will be educational traditions and cultural differences, pedagogical experience of interaction with strong-willed people, and educational contexts that facilitate the understanding of the values of an organized society.

A. Bergson, considering the concepts of morality, the moral obligation of a person to society, wrote: «Society, inherent in each of its members, has requirements, each of which, both large and small, expresses the integrity of its life force. Human society is a combination of free beings. The duties imposed by society, which allow it to exist, introduce into it an order that is simply similar to the steady order of the phenomena of life» (Bergson, 1994) [43, p.7].

We believe that the entire educational process should take into account the line of spiritual and moral education, and all educational influences should include aspects of the development of autonomous behavior. Without such education, pedagogical processes lose their meaning, and the contribution that the teacher makes to the preservation of the autonomy of the student's spirit would be significantly limited. In our opinion, it is wrong to separate the various elements that make up the entire complex process of managing autonomous behavior. And the purpose of this process is not only to reveal to teachers new knowledge about the autonomy of the spirit and its relationship with spirituality and morality, but also to promote the emergence of the practice of managing autonomous behavior. It should be noted that this knowledge has 
been collected in different cultures for centuries, and in addition to promoting the development of the autonomy of the spirit, knowledge about different cultural practices in different countries also provides teachers with different forms and methods of education. Thus, the management of autonomous behavior in modern pedagogy should include scientific knowledge, educational methods, technologies that contribute to the development of social and individual action, the organization of interaction in various cultural environments in such a way that both the material and intangible heritage of humanity, developed by free will, increases.

The crisis of spirituality around the world can be called a consequence of the crisis of scientific knowledge about spirituality, the questioning of religious doctrines by the consumer society, in which the education of public morality was fundamental. Having discarded the knowledge of religious figures about a man, about his spirit, many societies were not offered anything new in return, and therefore various occult practices began to be introduced into such societies, denying religion and its content of morality. To solve this problem, it is necessary to review the existing models of human behavior management in the consumer society and to acquire knowledge about the person in the spiritual, social and biological dimensions. Accordingly, this requires the creation of new methodologies, new pedagogical solutions that should take into account the new needs of the younger generation. Such approaches will help to develop an understanding of the management of autonomous behavior of the individual, and the development of new auxiliary materials should be aimed at helping teachers to support the participation of students in their spiritual and moral development, which will bridge the gap between the meanings of the life of an autonomous person and the content of education. That is why volitional or autonomous behavior management should be considered as an activity tool of the teacher, which is an integral part of the pedagogical process, which considers the ways of interaction, the needs of the individual and the needs of society.

Therefore, such a tool can help to raise students' awareness of their spiritual dimension, which will make it possible to identify the problems of their further selfdetermination and self-realization. This, in our opinion, can later lead to thinking about 
the need to change the behavior patterns that promote ataraxia (weak will, intemperance), as well as to understanding the need to reproduce the system of moral actions as an autonomous line of behavior. It should also be borne in mind that teachers also have their own values and personal interests, their own attitudes, they have their own way of understanding the autonomy of the spirit, and therefore, such experience should be studied and used later as a tool, the skillful use of which can make positive changes in the very life of each person and a new, organized society. 